\title{
What Is Trust? Ethics and Risk Governance in Precision Medicine and Predictive Analytics
}

\author{
Afua Adjekum, Marcello Ienca, and Effy Vayena
}

\begin{abstract}
Trust is a ubiquitous term used in emerging technology (e.g., Big Data, precision medicine), innovation policy, and governance literatures in particular. But what exactly is trust? Even though trust is considered a critical requirement for the successful deployment of precision medicine initiatives, nonetheless, there is a need for further conceptualization with regard to what qualifies as trust, and what factors might establish and sustain trust in precision medicine, predictive analytics, and large-scale biology. These new fields of 21 st century medicine and health often deal with the "futures" and hence, trust gains a temporal and ever-present quality for both the present and the futures anticipated by new technologies and predictive analytics. We address these conceptual gaps that have important practical implications in the way we govern risk and unknowns associated with emerging technologies in biology, medicine, and health broadly. We provide an in-depth conceptual analysis and an operative definition of trust dynamics in precision medicine. In addition, we identify three main types of "trust facilitators": (1) technical, (2) ethical, and (3) institutional. This three-dimensional framework on trust is necessary to building and maintaining trust in 21 st century knowledge-based innovations that governments and publics invest for progressive societal change, development, and sustainable prosperity. Importantly, we analyze, identify, and deliberate on the dimensions of precision medicine and large-scale biology that have carved out trust as a pertinent tool to its success. Moving forward, we propose a "points to consider" on how best to enhance trust in precision medicine and predictive analytics.
\end{abstract}

Keywords: precision medicine, trust, ethics, risk governance, predictive analytics, big data

\section{Introduction}

$\mathbf{P}$ RECISION MEDICINE refers to an emerging field for disease diagnostics, prevention, and targeted therapeutics that considers individual and population variability in genomes and other omics-es (proteome, metabolome, etc.), environment and lifestyles, with the aim of improving citizens' and patients' health and buttressing disease prevention and precision health interventions (Alessandrini et al., 2016; Florin and Escher, 2017). Moreover, precision medicine caters to both patients and healthcare professionals with respect to patient-centered care, customized patient-provider relationships and effective treatments (Adams and Petersen, 2016).

To a large extent, precision medicine is driven by three main concurrent trends: the increasing availability of heterogeneous large-scale databases from which novel patient aggregates evolve, advances in the characterization of medically relevant information, and novel computational tools for data analytics (Adams and Petersen, 2016; Collins and Var- mus, 2015). Exponential growth in data volume is a key feature of precision medicine.

Based on current rates of growth, researchers predict that healthcare data flow will reach the zettabyte $\left(10^{70}\right.$ bytes $)$ and, subsequently, the yottabyte scale $\left(10^{80}\right.$ bytes $)$ in the coming years (Raghupathi and Raghupathi, 2014). Collectively, these extensive databases-resulting mainly from human genome sequencing, proteomics, metabolomics, genomics, neuroimaging, and mobile health (m-health) data among others,- - work hand in hand with improvements in basic research to promote the concept of precision medicine (Collins and Varmus, 2015).

The potential inherent within precision medicine stems from its ability to transform current approaches to healthcare research and delivery. The Precision Medicine Initiative (PMI) commissioned by President Obama in 2015 illustrates this phenomenon. In the short term, it seeks to attain breakthroughs in oncological research with the aim of applying the lessons learned from these efforts to all other diseases in the future (Collins and Varmus, 2015). The PMI hopes to uncover

\footnotetext{
Health Ethics and Policy Lab, ETH Zurich, Zurich, Switzerland.
}

(C) Afua Adjekum, et al., 2017. Published by Mary Ann Liebert, Inc. This Open Access article is distributed under the terms of the Creative Commons Attribution Noncommercial License (http://creativecommons.org/licenses/by-nc/4.0/) which permits any noncommercial use, distribution, and reproduction in any medium, provided the original author(s) and the source are credited. 
relevant health information, however infinitesimal, by combining genomic and other omics information with clinical, lifestyle, as well as m-health data derived from large cohorts (Kaufman et al., 2016).

There is an increasing consensus that the notion of trust is paramount to derive maximum capabilities from precision medicine. A 2016 expert workshop held by the International Risk Governance Center (IRGC) identified trust as crucial to participant engagement in precision medicine since it thrives on biomedical data collection and sharing (Florin and Escher, 2017). In fact, at the onset of the PMI, the importance of public trust and privacy was spotlighted by the formation of an interagency working group (The Precision Medicine Initiative, 2015). Similarly, Mirnezami et al. (2012) have highlighted that precision medicine "will deeply affect public trust and the nature of the patient-clinician relationship," hence, "it will require unprecedented collaboration among healthcare stakeholders."

Although the salience of trust in precision medicine is a recurring theme, few studies have attempted to decipher the subject from a conceptual standpoint. Thus, this article seeks to underscore the salience of trust in precision medicine and to provide conceptual grounding and clarity by developing, at a minimum, a working definition and characterization of the trust dynamics. This is followed by identifying three main types of facilitators (technical, ethical, and institutional) necessary to building and maintaining trust in relation to precision medicine. Afterward, we deliberate on the dimensions of precision medicine that have carved out trust as a pertinent tool to its success. We conclude with some points to consider on how best to enhance trust in precision medicine.

\section{What is Trust?}

Although trust is a familiar concept that cuts across a spectrum of disciplines, there is a lack of consensus on its definition. Besides agreeing that trust is relational (occurs between people and or entities) and context specific, there is continual discourse among scholars on what constitutes trust (Gilson, 2003). Trust has been described as a three-place relation; whereby, "A" (trustor) trusts " $\mathrm{B}$ " (trustee) to fulfil "C" (task) (Baier, 1986). Overall, the concept of trust conveys the notion of risk and vulnerability on the part of a trustor.

Trust is often dichotomized into affective-based trust and cognitive-based trust. The former conveys the notion that trust stems from conventional norms of morality, which are upheld by the goodwill of others; whereas the latter perceives trust as a calculation or rational behavior that involves some form of risk analyses (Gilson, 2003). It is important to differentiate between trust and trustworthiness even though these two concepts are often used interchangeably. While trust is understood as an expectation of positive motives (Rousseau et al., 1998), trustworthiness is perceived as a characteristic that is projected onto an individual or group (Holm and Nystedt, 2010). O'Neill has famously brought attention to this important distinction arguing further that for trust to be established, trustworthiness needs to be demonstrated. In that respect, trust is a response to trustworthiness and we should aim to put "trustworthiness before trust"' (O'Neill, 2002).

Some scholars suggest that trust is neither a choice nor a behavior, but rather, a psychological state that is crucial to organizational life (Rousseau et al., 1998). Others maintain that encapsulated interests, rational predictions of another's behavior, as well as personality traits compel people to trust (Lang and Hallman, 2005). Nonetheless, reliance is at the core of trust even as it is assumed that a pure trust relationship exists when emotions such as gratitude, betrayal and resentment"reactive attitudes" — are expressed if expectations go unmet (Holton, 1994).

Trust is also at the helm of social cohesion even as it projects its existence —or lack thereof,- - on all aspects of social interactions. Social norms are said to shape individuals' beliefs and inherent in such beliefs is trust which in turn cultivates cooperation (Gilson, 2003). Within a trustworthy society, individuals balance into equilibrium both interpersonal trust (trust in individuals) and institutional trust (trust in the social system) (Gidman et al., 2012). But, although many scholars agree that these forms of trust rely on each other, they are yet to fully understand their underlying relationship (Gidman et al., 2012; Rousseau et al., 1998).

Generally, societies with optimal levels of trust witness lower transaction costs, and conflict aversion within organizations in addition to optimum cooperative behavior (Rousseau et al., 1998). Within the health system, trust facilitates patient satisfaction, adherence to treatments, health provider continuity, patient disclosures, and encourages access to health facilities (Gidman et al., 2012). It is important to note that trust cannot just be produced or generated, but must consistently be accumulated and reinforced by performance or experience (IRGC, 2005).

Trust is highly influenced by cultural, social, and political factors. This is especially true in the clinical setting, where due to unleveled power dynamics, healthcare providers (trustees) are oftentimes perceived to have absolute authority that patients will unlikely challenge (Chiu, 2011). This authority is partly a consequence of information asymmetry-unequal medical and scientific knowledge levels-between healthcare providers and patients (trustors), resulting in increased vulnerability for the latter. The vulnerability on the part of the trustors, furthermore, may expose them to possible exploitation by the trustee (Abelson et al., 2009; Calnan and Rowe, 2007).

In instances where there is low generalized trust (individuals' expectations of the trustworthiness of others) (OECD, 2017), mistrust and distrust are likely to thrive. Mistrust denotes unhealthy cynicism resulting from a prior breach of trust, whereas distrust comprises of healthy skepticism (Abelson et al., 2009). From this description, trust can be envisioned as a spectrum containing positive and negative peripheries: on the positive end sits "trust," with "mistrust" sitting on the negative end. As McAllister argues, "the amount of knowledge necessary for trust resides somewhere between total knowledge and total ignorance [...] Given total knowledge, there is no need to trust, and given total ignorance, there is no basis upon which to rationally trust" (McAllister, 1995).

As much as precision medicine relies on omics diagnostics to recognize disease patterns and subgroups, researchers relish voluntary data contributed from healthy individuals. Integral to this paradigm is the belief or expectation that personal data will not be used against the owner of the data and there will be a derivative public good (Bourzac, 2016). A study conducted by researchers at the U.S. National Institute of Health confirms this as individuals' willingness to participate in the PMI involves some risk and benefit analysis (Kaufman et al., 2016). 
Based on these evaluations, a working definition of trust in precision medicine could be: the willingness of a trustor to accept the potential risks involved in the sharing and further use of their personal data resulting from both optimism about the trustees' goodwill and interest in the public good.

While a trustor can be both an individual and a multiindividual entity (e.g., a corporation, research group, or other organization), in this article we are particularly concerned with the issue of trust from the perspective of the single individual toward the data initiatives for precision medicine. In fact, we consider the individual citizen to be the fundamental and atomic unit of trust.

It is also important to point out that trust dynamics are often multilayered and might follow chain reaction patterns (Fig. 1). In fact, when sharing data with a certain trustee $\mathrm{Y}$, a trustor $\mathrm{X}$ should not simply trust $\mathrm{Y}$, but also all actors that $\mathrm{Y}$ might further share X's data with. The longer the chain of trust, the harder it is for the individual to anticipate the risks and benefits of her voluntary data contribution as well as to assess the trustworthiness of all trustees involved. This problem is exacerbated by the fact that, in many circumstances, the indi- vidual citizen might have only partial—if any-knowledge about actors beyond the first trustee.

It is noteworthy in this context that the 21 st century science initiatives such as PMI are large network-driven sciences, not to mention large-scale databases and Big Biology. Networks can bring up to scale scientific discoveries and innovation from a pragmatic standpoint, but can also exert network power and authority on the atomic unit of trust, the individual and her/his agency, if networks, their powers and attendant authority, entrenchment, and group thinking are not kept in check (Bourdieu, 1986; Bourdieu and Wacquant, 1992; Haraway, 1988). Indeed, feminist theorists and scholars have underscored the multipronged, underappreciated and unpredictable ways in which network power and hierarchy shape persons and their agency; these are particularly relevant in 21st century Big Science initiatives (Bourdieu, 1986; Foucault, 1980; Haraway, 1988).

These considerations show that interpersonal (e.g., between the data generator and the data aggregator) trust in precision medicine also has implications for the notion of agency, that is, the manifestation of the capacity to act. For example, the

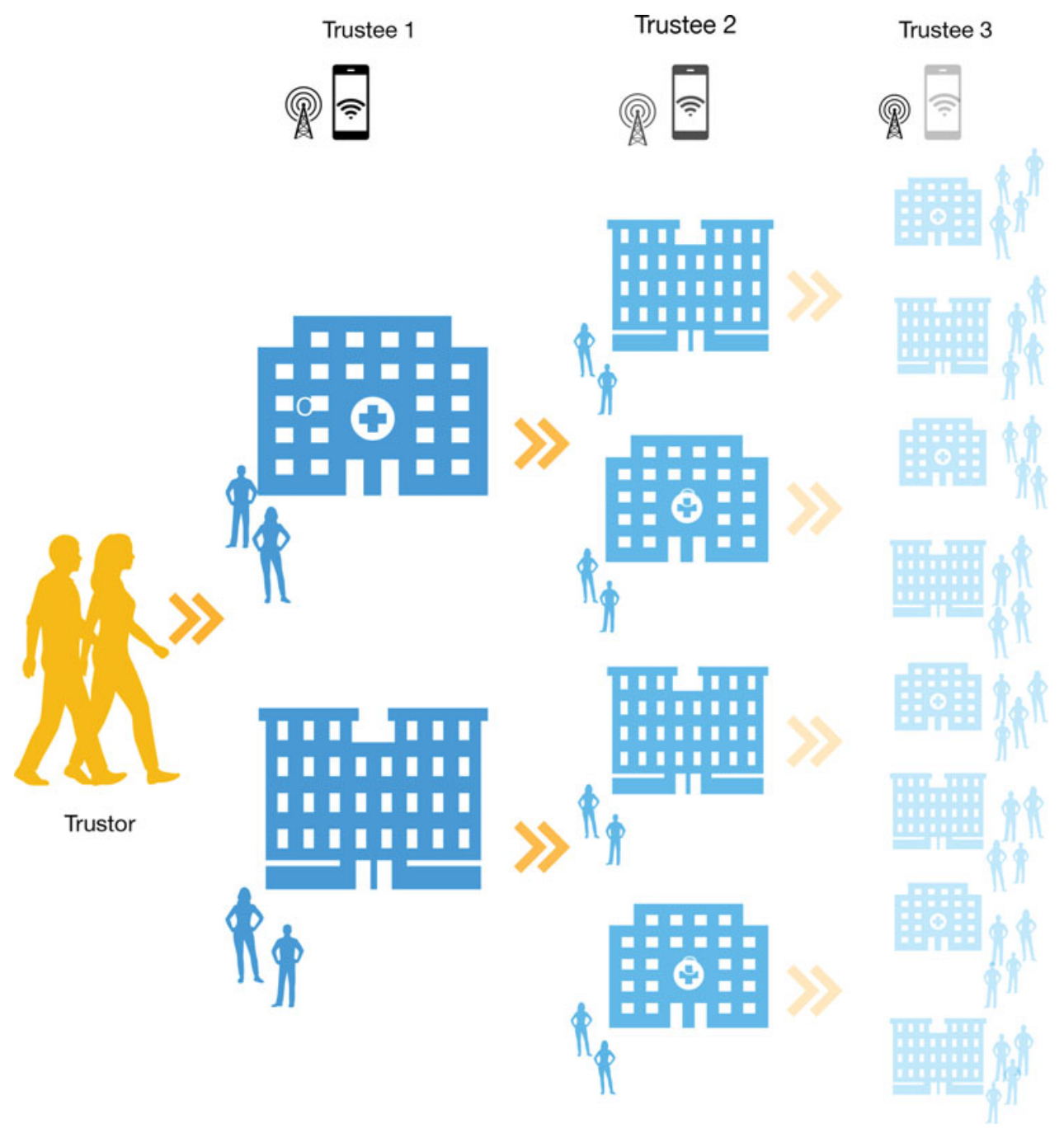

FIG. 1. The complex data sharing relationship between a trustor and relevant trustees (either individuals or institutions). The arrows indicate the various trust relationships occurring at different phases of the data-sharing process. The fading represents diminishing trustors' knowledge about data-sharing mechanisms and actors. 
absence of adequate knowledge about the entire data processing cycle, undermines an agent's (person's) capacity to perform informed risk-benefit analyses. This risk is particularly relevant in the context of precision medicine activities involving Big Data and predictive analytics, since they implicate increased informational complexity and, in many circumstances, reduced algorithmic transparency.

Nonetheless, we reject the idea that interpersonal trust and personal autonomy are mutually incompatible in the context of precision medicine. As Oshana (2014) has argued in relation to healthcare provision, "attitudes of interpersonal trust" are not simply "consistent with autonomous agency," but could even "promote and reinforce autonomous agency" provided that the patient has "sound reason to place her trust in the complex of institutions that make possible the provision of health care, including the hospital administrators, the insurance industry, and the legal and judicial system". Similarly, we argue that, in the specific context of precision medicine, the individual data donor has sound reason to place her trust in the complex of institutions that make possible the collection and analysis of health data. For this condition to be fulfilled, however, there is a need for identifying and promoting some facilitators of trust.

\section{Facilitators for Sustaining Trust}

The drivers for building trust in precision medicine can be categorized into three: (1) technological innovation, (2) ethical and sociocultural values, and (3) institutional practices and governance measures.

Technological innovation is a viable means of facilitating accountability relating to data sharing, quality, and integrity. A typical example is the blockchain technology: which refers to a digital ledger made up of linked peer-to-peer transaction blocks on which unalterable records are shared. The features of the blockchain makes it well suited to address the disjointed nature of health-related records by eliminating the need to rely on third parties for their security (Krawiec et al., 2016). Consequently, the blockchain stands to increase health data privacy, security, as well as interoperability (IRGC, 2017).

Differential privacy is another technological solution that can minimize the risks associated with the use of sensitive data. Differential privacy mechanisms aim to uphold the analysis of databases containing confidential information by, among others, minimizing the risks associated with the reidentification of anonymized data (Dwork and Roth, 2014). In ensuring a high standard of data anonymization-by shrinking the risks associated with being reidentified with publicly available data for instance,--individuals are more likely to participate in PMIs.

It is crucial to recognize that technology alone cannot ensure that patients and other stakeholders place their full trust in the advances that precision medicine has to offer. Rather, it can help build and sustain trust by reassuring participants of the privacy and security of their sensitive data. Moreover, technology as a tool to help build and sustain trust in precision medicine would only attain optimum results if it accompanies sound medical analysis coupled with meaningful and equitable relationships with patients and citizens.

Perceiving ethical or sociocultural values as facilitators to trust in precision medicine stems from experiences with trust in similar conditions. The relevance of transparency and public trust in novel biomedical research has been echoed ever since major strides were accomplished in recombinant DNA and beyond (Baltimore et al., 2015). With respect to precision medicine, it is essential to prioritize transparency that ranges from healthcare delivery, research, as well as institutional management practices.

Preferred forms of consent, data sharing in addition to the motivations for participation must be constantly assessed to alleviate participants' concerns (Kaufman et al., 2016). In prioritizing the participant (trustor), the researcher or health personnel (trustee) is better able to relay their proficiency to undertake the tasks assigned them and likely reinforce their goodwill. This matters as the information sheets and consent forms that are disbursed during research projects for instance, have been shown to merely serve as "symbolic tokens." Instead, participants' "faith" in the premise for the project and an assurance of minimal risks are what motivate them to participate (Carter et al., 2015).

Public engagement strategies based on communication are another ample ethical or sociocultural values tool. The role of communication in public engagement strategies is understood as a two-way dialog between relevant stakeholders and the public. Good practices in risk communication help stakeholders to make informed choices about matters of concern to them and hence create mutual trust (IRGC, 2008). The absence of dialog-based communication regarding the roles and expectations of both a trustor and trustee results in misplaced trust. Misplaced trust originates from disappointment rather than deliberate moral malevolence (Hawley, 2015).

Stakeholder engagement on a broad scale facilitates better decision making (IRGC, 2014). By involving all of the entities to whom precision medicine matters, the motivations behind individuals' values and interests are identified and prioritized. A study conducted by Kaufman et al. (2016) reinforces the value of consistent stakeholder engagement prior and during PMIs even as $30 \%$ of survey participants after learning of the potential risks and benefits of the PMI cohort study altered their eagerness to participate.

Institutional practices and governance are linked to social cohesion making them critical trust facilitators. Gilson (2003) maintains that trust in institutions (both public institutions and other social organizations, including private ones) bolster the social order by ensuring interpersonal trust, which in turn strengthens generalized trust. Hence, management practices and decisions do not only matter to those said institutions but also to society as a whole. Equity, fairness, and a general sense of prioritizing the public good at the institutional or governance levels all stand to foster trust in precision medicine and society as a whole.

Public institutions that aim to foster trust must prioritize their perceived competence and values among the public. Competence is the capacity to undertake an intended directive, while values foretell the intentions and ethics of an institution (OECD, 2017). In as much as competence and values matter, however, it is crucial to bear in mind that individuals' overall trust judgments regarding an organization are equally influenced by transparency and openness, public interest (organizational aim to tackle bias), and honesty (truthful about risk) (Lang and Hallman, 2005).

Governance models include a variety of options such as incentives, frameworks, initiatives, best practices, or soft law (IRGC, 2008). With effective governance approaches, institutions are more equipped to achieve a common basis for 
measurable, transparent, and comparable quality standards. In the case of precision medicine, it stands to assure the public of the standardization of PMIs. An example of governance relevant for precision medicine is the Committee of Ministers of the Council of Europe Recommendation CM/ Rec (2016)6, which assesses legal guidelines for research on biological materials of human origin in research projects within Europe. The Committee safeguards the fundamental rights of individuals from whom biological materials are obtained, stored, and used (Council of Europe, 2016).

Schlesinger and Gray (2016) argue that trustworthiness is never guaranteed in healthcare due to the challenges involved with measuring trust within this setting, combined with the ubiquity of provider discretion. Yet, studies show that experts and organizations can favorably impact public perception which in turn helps to advance efforts to build and maintain overall trust within a society. As a result, efforts to build and sustain trust in precision medicine must recognize and acknowledge this paradigm moving forward.

\section{How to Achieve Trust in Precision Medicine?}

Precision medicine depends on the aggregation of a broad quantity and variety of patient data. Standard health data sources such as medical records, genomics, immunization records, are giving way to include, among others, data from self-tracking devices, store transactions, wellness, and social media (Vayena and Blasimme, 2017). For these heterogeneous data to be available (hence, for such data aggregation to be possible), researchers and clinicians need to rely on voluntary data contributions from individuals. Since familiarity is vital to building and maintaining trust, it will take some time to ensure public acquaintance with these novel health data sources and stakeholders. In addition, this increasing breadth and variety of data sources will progressively require collaborative efforts between several disciplines as well as novel stakeholders.

Furthermore, precision medicine relies heavily on sensitive patient data that may be made available to new and previously unfamiliar actors in healthcare. Reports show that health data are currently more valuable than credit card information; increasing health organizations' cyber incidents from $20 \%$ in 2009 to $40 \%$ in 2013 (Humer and Finkle, 2014). This calls for an increased need to ensure optimum health data privacy and security. Perhaps, bolstering regulation concerning negligence with health data might be a feasible way to combat unnecessary health data breaches. However, increased regulations may also adversely affect institutional trust (Calnan and Rowe, 2007).

It is vital to consider these elements in the future to avoid repeating past and even novel mistakes. In 2012, under the Health and Social Care Act, the National Health Service (NHS) proposed the care.data initiative. Despite meeting the legal requirements to exploit medical records for research, care.data floundered (Carter et al., 2015; Sterckx and Cockbain, 2014). Its failure has been attributed to three main factors: (1) ambiguity of derivative public good, (2) faulty warrants of trust, as well as (3) uncertainties surrounding the duties of general practitioners. Indeed, it was not inadequate publicity that derailed care.data, but rather, an absence of faith, a lack of transparency compounded by ambivalence about the risks involved (Carter et al., 2015).

More recently the NHS England had another controversial collaboration with Google's DeepMind. In this case, DeepMind received access to millions of identifiable patient data to develop applications that would support patients with kidney disease. An investigation of the data sharing agreement between DeepMind and the NHS by the U.K.'s National Data Guardian concluded that this arrangement took place on an inappropriate legal basis (Powles and Hodson, 2017; Revell, 2017).

The two controversial and contested cases above highlight problems that can easily emerge in the sensitive space of patient data. They also illustrate some specific points of consideration that can facilitate our thinking of how best to promote trust in the data-rich environment of precision medicine. Below is a preliminary list of suggestions:

First, we identify a need for the widespread implementation of technical facilitators of trust in PMIs. These include, but do not necessarily restrict to, blockchain and differential

Table 1. A Three-Dimensional Approach and Points to Consider in Relation to Trust Facilitators in PReCision Medicine

\begin{tabular}{|c|c|c|c|}
\hline $\begin{array}{l}\text { Type of trust } \\
\text { facilitator }\end{array}$ & Points to consider & Actions (normative) & Examples \\
\hline $\begin{array}{l}\text { Dimension 1: } \\
\text { technical }\end{array}$ & $\begin{array}{l}\text { Value of } \\
\text { sensitive data }\end{array}$ & $\begin{array}{l}\text { Disintermediating data from hosts } \\
\text { Increasing transparency and process integrity } \\
\text { Reducing vulnerability to data leakage and } \\
\quad \text { malicious attacks }\end{array}$ & $\begin{array}{l}\text { Differential privacy } \\
\text { Blockchain } \\
\text { Encryption }\end{array}$ \\
\hline $\begin{array}{l}\text { Dimension 2: } \\
\text { ethical/ } \\
\text { sociocultural }\end{array}$ & $\begin{array}{l}\text { Unfamiliarity of } \\
\text { the public with } \\
\text { novel healthcare } \\
\text { actors }\end{array}$ & $\begin{array}{l}\text { Prioritizing privacy, transparency, and fairness } \\
\text { Promoting public engagement and awareness } \\
\text { Unambiguously anticipating public good } \\
\text { Identifying reliable warrants of trust }\end{array}$ & $\begin{array}{l}\text { Privacy protection } \\
\text { Accountability mechanisms } \\
\text { Transparency of process } \\
\text { Fair benefit sharing plans } \\
\text { Public engagement }\end{array}$ \\
\hline $\begin{array}{l}\text { Dimension 3: } \\
\text { institutional }\end{array}$ & $\begin{array}{l}\text { Susceptibility to } \\
\text { data breaches }\end{array}$ & $\begin{array}{l}\text { Establishing international networks of } \\
\text { researchers } \\
\text { Creating trustworthy platforms for } \\
\text { exchange } \\
\text { Defining a clearer ethical process for precision } \\
\text { medicine research }\end{array}$ & $\begin{array}{l}\text { Standardized guidelines } \\
\text { Ethical review recommendations }\end{array}$ \\
\hline
\end{tabular}


privacy technology. On the long term, the successful implementation of these and other privacy-enhancing techniques (e.g., encryption) might enhance existing security protocols and models in terms of both format and structure and "add additional layers of security and trust" (Versel, 2017). In fact, these technologies potentially allow to disintermediate data from hosts, increase transparency and process integrity, increase the ability to withstand data leakage and malicious attacks, and ultimately empower trustors. Therefore, this is likely to have a positive effect not only on the management of healthcare data but also on their largescale collection as well as on the social dissemination of scientific findings.

Concurrently, this technical transition should be coordinated with the responsible promotion of ethical and sociocultural facilitators. Ethical values such as privacy, transparency, and fairness are likely to be codeterminants of trust at both the individual and collective level; hence, should be prioritized to maximize the benefits of precision medicine in an ethically sustainable manner. Analogously, public engagement and awareness-raising activities should be incentivized to facilitate competent decision making within the precision medicine ecosystem, especially from the perspective of the trustor-trustee relationship. As the failure of the care.data initiative indicates, stakeholder engagement should be sustained through an unambiguous anticipation of derivative public good, reliable warrants of trust, and a clear specification of the duties of health professionals.

Finally, calibrated institutional interventions and governance solutions should be advanced to enhance trust in precision medicine and orient research for the public good. A recent national example, the Swiss Personalized Health Network (SPHN) is founded on the principles of responsible data processing, namely respect for persons, privacy, data fairness, and accountability (ELSI Advisory Group, 2017). Ideally, these networks of researchers should be established not only at the national level but also internationally.

An initiative taking this international approach (although focusing on genomics) is the Global Alliance for Genomics and Health (www.ga4gh.org). Platforms may also facilitate dialog and information sharing not only between researchers but also between the precision medicine research and other societal actors. Finally, to promote and sustain trust in precision medicine research, institutional review board (IRBs) and other deliberative assemblies should clearly define effective and reliable ethical review processes for precision medicine research. Table 1 summarizes our analysis of trust facilitators and the subsequent points of consideration.

\section{Conclusions and Outlook}

Trust is essential in precision medicine, predictive analytics and large-scale biology. These new fields of 21st century medicine and health often deal with the "futures" and hence, trust gains a temporal and ever-present quality for both the present and the futures anticipated by predictive analytics. However, the characterization of trust dynamics in this rapidly evolving field of medicine is often affected by uncertainty and conceptual variability.

In this article, we provide a definition of trust and a detailed characterization of trustor-trustee dynamics in precision medicine. We highlight that trust is necessary for facilitating partic- ipants' involvement in PMIs; hence, it is a catalyzer for socially responsible scientific advancement in this field. Technical solutions, ethical, and sociocultural values as well as effective governance measures were all identified as facilitators of trust in precision medicine. Based on this analysis and learning from previous PMIs, we proposed a minimal set of recommendations aimed at minimizing the risks associated with the sharing of sensitive data, increasing transparency, empowering trustors, and augmenting public trust in precision medicine.

\section{Acknowledgments}

This project was funded by grants from the Swiss National Science Foundation (SNSF) to Professor Vayena (PP000P3157556; 4075210-167223). This article was developed as a background document for an expert workshop on Trust in Precision Medicine-A Risk Governance Perspective (Geneva, Switzerland, November 23-24, 2017) organized by IRGC in collaboration with the SPHN, ETH Zurich, EPFL, CHUV, and University of Geneva. The authors thank Marie-Valentine Florin and Marcel Bürkler at the IRGC, Lausanne, for their invaluable input to earlier versions of the article.

\section{Author Disclosure Statement}

The authors declare that no conflicting financial interests exist.

\section{References}

Abelson J, Miller FA, and Giacomini M. (2009). What does it mean to trust a health system?: A qualitative study of Canadian health care values. Health Policy 91, 63-70.

Adams SA, and Petersen C. (2016). Precision medicine: Opportunities, possibilities, and challenges for patients and providers. J Am Med Inform Assoc 23, 787-790.

Alessandrini M, Chaudhry M, Dodgen TM, and Pepper MS. (2016). Pharmacogenomics and global precision medicine in the context of adverse drug reactions: Top 10 opportunities and challenges for the next decade. OMICS 20, 593-603.

Baier A. (1986). Trust and antitrust. Ethics 96, 231-260.

Baltimore D, Berg P, Botchan M, et al. (2015). A prudent path forward for genomic engineering and germline gene modification. Science 348, 36-38.

Bourdieu P. (1986). The forms of capital. In: Handbook of Theory and Research for the Sociology of Education. Richardson JG, ed. New York, NY: Greenwood, 241-258.

Bourdieu P, and Wacquant L. (1992). Invitation to Reflexive Sociology. Chicago, IL: Chicago University Press.

Bourzac K. (2016). Power to the patients. Nature 537, S66-S68.

Calnan M, and Rowe R. (2007). Trust and health care. Sociol Compass 1, 283-308.

Carter P, Laurie GT, and Dixon-Woods M. (2015). The social license for research: Why care.data ran into trouble. J Med Ethics 41, 404-409.

Chiu YC. (2011). Probing, impelling, but not offending doctors. Qual Health Res 21, 1658-1666.

Collins FS, and Varmus H. (2015). A new initiative on precision medicine. N Engl J Med 372, 1-3.

Council of Europe. (2016). Recommendation CM/Rec(2016)6 of the Committee of Ministers to member States on research on biological materials of human origin.

Dwork C, and Roth A. (2014). The algorithmic foundations of differential privacy. Found Trends Theor Comput Sci 9, 211-407. 
ELSI Advisory Group. (2017). Ethical Framework for Responsible Data Processing in the Swiss Personalized Health Network, Bern.

Florin M-V, and Escher G. (2017). A Roadmap for the Development of Precision Medicine. Lausanne: EPFL International Risk Governance Center (IRGC).

Foucault M. (1980). Power/Knowledge: Selected Interviews and Other Writings, 1972-1977. Gordon C, ed. New York: Pantheon Books.

Gidman W, Ward P, and McGregor L. (2012). Understanding public trust in services provided by community pharmacists relative to those provided by general practitioners: A qualitative study. BMJ Open 2, e000939.

Gilson L. (2003). Trust and the development of health care as a social institution. Soc Sci Med 56, 1453-1468.

Haraway D. (1988). Situated knowledges: The science question in feminism and the privilege of partial perspectives. Fem Stud 14, 575-599.

Hawley K. (2015). Trust and distrust between patient and doctor. J Eval Clin Pract 21, 798-801.

Holm HJ, and Nystedt P. (2010). Collective trust behavior. Scand J Econ 112, 25-53.

Holton R. (1994). Deciding to trust, coming to believe. Australas J Philos 72, 63-76.

Humer C, and Finkle J. (2014). Your Medical Record Is Worth More to Hackers Than Your Credit Card. Reuters. https:// www.reuters.com/article/us-cybersecurity-hospitals/yourmedical-record-is-worth-more-to-hackers-than-your-creditcard-idUSKCNOHJ21I20140924

International Risk Governance Council. (2008). An introduction to the IRGC Risk Governance Framework, Geneva.

International Risk Governance Council. (2005). White paper on Risk Governance Towards and Integrative Approach, Geneva. International Risk Governance Center IRGC. (2017). Governing risks and benefits of distributed ledger technology applications in insurance, medical and institutional governance, and with a focus on privacy and security issues, Lausanne.

International Risk Governance Council. (2014). Stakeholder engagement resource guide for developing and implementing science-based stakeholder involvement research, policy, strategies, and practices, Lausanne.

Kaufman DJ, Baker R, Milner LC, Devaney S, and Hudson KL. (2016). A survey of U.S adults' opinions about conduct of a nationwide Precision Medicine Initiative ${ }^{\circledR}$ cohort study of genes and environment. PLoS One 11, e0160461.

Krawiec RJ, Housman D, White M, et al. (2016). Blockchain: Opportunities for Health Care. Deloitte.

Lang JT, and Hallman WK. (2005). Who does the public trust? The case of genetically modified food in the United States. Risk Anal 25, 1241-1252.

Mcallister DJ. (1995). Affect- and cognition-based trust as foundations for interpersonal cooperation in organizations. Acad Manag 38, 24-59.

Mirnezami R, Nicholson J, and Darzi A. (2012). Preparing for precision medicine. N Engl J Med 366, 489-491.

O'Neill O. (2002). A Question of Trust: The BBC Lectures 2002. Cambridge University Press. http://www.efc.be/humanrights-citizenship-democracy/trust-trustworthiness-transparency/
OECD. (2017). Trust and Public Policy: How Better Governance Can Help Rebuild Public Trust, OECD Public Governance Reviews. Paris: OECD Publishing.

Oshana M. (2014). Trust and autonomous agency. Res Philosophica 91, 431-447.

Powles J, and Hodson H. (2017). Google DeepMind and healthcare in an age of algorithms. Health \& Technol 1-17. https:// doi.org/10.1007/s12553-017-0179-1

Raghupathi W, and Raghupathi V. (2014). Big data analytics in healthcare: Promise and potential. Health Inf Sci Syst 2, 1-10.

Revell T. (2017). Google DeepMind NHS Data Deal was "legally inappropriate." New Scientist. https://www.newscientist.com/ article/2131256-google-deepmind-nhs-data-deal-was-legallyinappropriate/

Rousseau DM, Sitkin SB, Burt RS, and Camerer C. (1998). Introduction to special topic forum: Not so different after all : A Cross-discipline view of trust. Acad Manag Rev 23, 393-404.

Schlesinger M, and Gray BH. (2016). Incomplete markets and imperfect institutions: Some challenges posed by trust for contemporary health care and health policy. J Health Polit Policy Law 41, 717-742.

Sterckx S, and Cockbain J. (2014). The UK national health service's 'innovation agenda': Lessons on commercialisation and trust. Med Law Rev 22, 221-237.

The Precision Medicine Initiative. (2015). Precision Medicine Initiative: Privacy \& Trust Principles. Washington D.C.: White House.

Vayena E, and Blasimme A. (2017). Biomedical big data: New models of control over access, use and governance. J Bioeth Inq [Epub ahead of print]. DOI: 10/1007/s11673-017-9809-6

Versel N. (2017). Blockchain Eyed for Boosting Data Security, Trust in Precision Medicine. Genomeweb.

Address correspondence to: Effy Vayena, PhD

Health Ethics and Policy Lab ETH Zurich

Auf der Mauer 17 Zurich 8001

Switzerland

E-mail: effy.vayena@hest.ethz.ch

\section{Abbreviations Used}

$\mathrm{CM} / \mathrm{Rec}=$ Council of Europe Recommendation DNA $=$ deoxyribonucleic Acid

ELSI $=$ Ethical, Legal, and Social Implications

IRB $=$ Institutional Review Board

IRGC = International Risk Governance Center

NHS $=$ National Health Service

OECD = Organization for Economic Co-operation and Development $\mathrm{PMI}=$ Precision Medicine Initiative SNSF $=$ Swiss National Science Foundation SPHN $=$ Swiss Personalized Health Network 